\title{
Efficacy of four hollow nail rhombic fixation for the treatment of patients with femoral neck fractures: A protocol of systematic review and meta-analysis
}

Qing-hui Ji

Southern Medical University Nanfang Hospital

Yu Xue

Second Affiliated Hospital of Jiamusi University

Jie Miao

Handan Central Hospital

Zhi-xin Reng

Handan Central Hospital

Yu-fei Yuan

Handan Central Hospital

Yan-bao Li ( $\nabla$ yanbaoli2001@yeah.net)

Handan Central Hospital

\section{Protocol}

Keywords: Femoral neck fractures, four hollow nail rhombic fixation, efficacy, safety

Posted Date: May 18th, 2020

DOl: https://doi.org/10.21203/rs.3.rs-27662/v1

License: (9) (i) This work is licensed under a Creative Commons Attribution 4.0 International License.

Read Full License 


\section{Abstract}

Background: This study aims to assess the efficacy of four hollow nail rhombic fixation (FHNRF) for the treatment of patients with femoral neck fractures (FNF).

Methods : A literature search in MEDLINE, Scopus, Web of Science, EMBASE, Cochrane Library, ProQuest, Thesis and Dissertation Catalog, Cumulative Index to Nursing and Allied Health Literature, and China National Knowledge Infrastructure will be performed from inception through February 29, 2020. This study will not apply limitations to the language and publication date. All potential randomized controlled trials (RCTs) that identify the efficacy and safety of FHNRF for the treatment of patients with FNF. Two contributors will separately examine searched records, extract essential data, and assess study quality using Cochrane risk of bias tool. Any opposition between two authors will be settled by a third contributor. We will employ RevMan 5.3 software for statistical analysis.

Discussion : This study will summarize high quality RCTs to assess the efficacy and safety of FHNRF for the treatment of patients with FNF. It will help to determine whether or not FHNRF is effective and safety for the treatment of patients with FNF. Systematic review registration CRD42020168378.

\section{Background}

Femoral neck fractures (FNF) is a common injury in the emergency visits, especially in elderly population [1-3]. It is estimated that the incidence of hip factures is approximately 6 million by 2050 worldwide [45], and FNF accounts for about $50 \%$ of all hip fractures [6-7]. It is associated with impaired mobility, loss of function, mortality and morbidity [8-11]. The optimal management of FNF is surgery [12].

Studies suggested that four hollow nail rhombic fixation (FHNRF) can be utilized for the treatment of FNF [13-20]. However, no systematic review is published on investigating the efficacy and safety of FHNRF for the treatment of FNF. This study aims to determine the efficacy and safety of FHNRF in the treatment of FNF by assessing the quality of the available evidence. The specific question addressed by this study: is FHNRF effective and safe in the treatment of patients with FNF?

\section{Methods And Analysis}

\section{Study registration}

This study has been registered on PROSPERO (CRD42020168378). We have reported it following the guideline of Preferred Reporting Items for Systematic Reviews and Meta-Analysis Protocol statement [21-22].

\section{Eligibility criteria for study selection}




\section{Types of study}

All randomized controlled trials (RCTs) that appraised the efficacy and safety of FHNRF for the treatment of patients with FNF will be included. We will exclude all other studies, such as laboratory studies, case report, case series, review, and non-clinical trial.

\section{Types of participant}

All patients who were diagnosed with FNF will be included, in spite of their characteristics, and duration and severity of FNF.

\section{Types of intervention}

\section{Interventions}

All patients in the interventional group received FHNRF as their therapy.

\section{Comparators}

Studies comparing any other treatments, such as partial hip replacement, and total hip replacement will be included in this study.

\section{Types of outcome measurement}

The primary outcome is pain intensity, which has been assessed by any relevant pain scales, such as Visual Analogue Scale.

The secondary outcomes are stiffness and physical function (as examined by any associated index, such as Western Ontario and McMaster Universities Osteoarthritis Index); and quality of life (as assessed by any related scales, such as 36-Item Short Form Health Survey), and adverse events.

\section{Literature search}

We will systematically and comprehensively conduct searches in MEDLINE, Scopus, Web of Science, EMBASE, Cochrane Library, ProQuest, Thesis and Dissertation Catalog, Cumulative Index to Nursing and Allied Health Literature, and China National Knowledge Infrastructure from inception through February 29, 2020 with no restrictions to language and publication date. We will consider all potential RCTs that explored the efficacy and safety of FHNRF for the treatment of patients with FNF. The full search strategy 
for MEDLINE is displayed (table 1), and we will adapt similar search strategies for other electronic databases.

We will identify other sources to avoid losing potential studies, such as dissertations/thesis, conference proceedings and reference lists of included RCTs.

\section{Study selection}

Two examiners will independently evaluate the titles and abstracts of the found literatures during the searches for potential inclusion. All irrelevant and duplicated studies will be removed. Potential relevant articles will be carefully read in full by the same two examiners. The articles that meet all eligibility criteria will be included. Different views will be discussed in consensus conferences with the help of a third examiner. The results of study selection will be presented in a flow diagram.

\section{Data extraction and management}

Two examiners will independently extract data from eligible studies. The extracted information includes trial setting, trial characteristics (e.g. first author, time of publication, et el), research design, details of intervention and comparator, eligibility criteria, outcomes, patient characteristics (e.g. sample size, sex, age, co-morbidities, et al), results, conclusion and conflict of interest. Any discrepancies will be solved through discussion with a third examiner.

\section{Missing data dealing with}

If there is unclear or missing data, primary authors will be contacted to request it by email or telephone. In case of unavailable data, we will analyze present data using an intention-to-treat analysis.

\section{Risk of bias assessment}

Two examiners will separately identify risk of bias for each eligible study using Cochrane Risk of Bias Tool. It assesses risk of bias through 7 aspects and each one is graded as low, unclear or high risk of bias. Opposite opinions will be arbitrated by a third examiner through discussion.

\section{Statistical analysis}

\section{Data synthesis}


We will place RevMan 5.3 software to analyze extracted data, and carry out a meta-analysis whenever it is possible. We will estimate the pooled treatment effects of dichotomous data as risk ratio and $95 \%$ confidence intervals (Cls), and those of continuous data as weighted mean difference or standardized mean difference and $95 \%$ Cls. We will check statistical heterogeneity across RCTs by $l^{2}$ test. $l^{2} \leq 50 \%$ exerts little statistical heterogeneity, and a fixed-effects model will be applied. $I^{2}>50 \%$ indicates distinct heterogeneity, and a random-effects model will be employed. A subgroup analysis will be conducted to test possible reasons of apparent heterogeneity. If there is still evident heterogeneity after subgroup analysis, we will conduct a narrative summary.

\section{Subgroup analysis}

We will carry out a subgroup analysis to explore the sources of obvious heterogeneity based on the different types of study and patient characteristics, interventions and comparators, and outcomes.

\section{Sensitivity analysis}

We will perform a sensitivity analysis to test the robustness of study findings based on the methodological weaknesses and missing data.

\section{Publication bias}

We will conduct a funnel plot and Egger's test to investigate the publication biases when more than 10 RCTs are included.

\section{Grading quality of evidence}

Two examiners will separately appraise the quality of evidence for major outcomes by Grading of Recommendations Assessment, Development, and Evaluation System approach [23-24]. Any conflicts will be cleared up by discussion with a third examiner, and a final consensus will be reached.

\section{Dissemination}

We will publish this study via a peer-reviewed journal.

\section{Discussion}

FNF is a common disorder in the elderly population, which often brings very poor quality of life in patients with FNF. Currently, FHNRF approach is used for the treatment of patients with FNF. In spite of the clinical and experimental support, the efficacy and safety of FHNRF for FNF have not been fully validated and 
evaluated. In addition, no systematic review and meta-analysis regarding it has been done. Therefore, this study aims to evaluate the efficacy and safety of FHNRF for the treatment of patients with FNF. Its findings may supply evidence for the reference of clinical practice and health-related policy maker.

\section{Abbreviations}

FHNRF, four hollow nail rhombic fixation; FNF, femoral neck fractures; RCTs, randomized controlled trials; Cls, confidence intervals.

\section{Declarations}

Ethics approval and consent to participate: Not applicable

Consent for publication: Not applicable

Availability of data and material: Data sharing is not applicable to this article as no datasets were generated or analyzed during the current protocol

Competing interests: Not applicable.

Funding:Not applicable.

Authors' contributions:QHJ and YBL conceived the study. ZXR and YFY contributed with the clinical background and expertise. QHJ, YX, JM, and YBL contributed with the analytical plan and the bias assessment approach. ZXR and YFY performed the literature search plan. QHJ, YX, JM, YFY, and YBL drafted the protocol. All authors revised the protocol and approved the final version. YBL supervised the study

Acknowledgements: Not applicable.

Authors'information: Qing-hui Ji, qinghui9652@yeah.net

Yu Xue,yuxue201103@yeah.net

Jie Miao, shuirao92@163.com

Zhi-xin Reng, rupeeshgnwj@outlook.com

Yu-fei Yuan, nonglu607419784@126.com

Yan-bao Li,yanbaoli2001@yeah.net

\section{References}


1.Barquet A, Giannoudis PV, Gelink A. Femoral neck fractures after removal of hardware in healed trochanteric fractures. Injury 2017; 48(12):2619-24.

2.Frihagen F. On the diagnosis and treatment of femoral neck fractures. Acta Orthop Suppl 2009; 80(335):1-26.

3.Wei L, Sun JY, Wang Y, Yang X. Surgical treatment and prognosis of acetabular fractures associated with ipsilateral femoral neck fractures. Orthopedics 2011;34(5):348.

4.De Laet CE, Pols HA. Fractures in the elderly: epidemiology and demography. Baillieres Best Pract Res Clin Endocrinol Metab 2000; 14(2):171-9.

5.Johnell O, Kanis JA. An estimate of the worldwide prevalence and disability associated with osteoporotic fractures. Osteoporos Int 2006; 17(12):1726-33.

6.Varacallo MA, Fox EJ. Osteoporosis and its complications. Med Clin North Am 2014; 98(4):817-31.

7.Varacallo MA, Fox EJ, Paul EM, Hassenbein SE, Warlow PM. Patients' response toward an automated orthopedic osteoporosis intervention program. Geriatr Orthop Surg Rehabil 2013; 4(3):89-98.

8.Cooper C, Campion G, Melton LJ $3^{\text {rd }}$. Hip fractures in the elderly: a world-wide projection. Osteoporos Int 1992; 2:285-289.

9. Cooper C, Atkinson EJ, Jacobsen SJ, O'Fallon WM, Melton LJ $3^{\text {rd }}$. Population-based study of survival after osteoporotic fractures. Am J Epidemiol 1993; 137:1001-5.

10.Kannus P, Parkkari J, Sievänen H, Heinonen A, Vuori I, Järvinen M. Epidemiology of hip fractures. Bone 1996; 18(1 Suppl): 57S-63S.

11.Cummings SR, Black DM, Nevitt MC, et al. Bone density at various sites for prediction of hip fractures. Lancet 1993; 341(8837):72-5.

12.Parker MJ, Gurusamy KS, Azegami S. Arthroplasties (with and without bone cement) for proximal femoral fractures in adults. Cochrane Database Syst Rev 2010; 6:CD001706.

13.Yin H, Zhou EC, Pan ZJ, Chen G, Jiang H. Finite element analysis of 4 hollow nails and 3 hollow nails combined with support plate internal fixation for Pauwels type $\otimes$ femoral neck fractures. China Tissue Engineering Research 2019; 23 (32): 5133-7.

14.Wang Y, Liu ZI, Yin T, et . Biomechanical study on the treatment of femoral neck fracture with internal fixation using finite element method. Chinese Integrative Medicine Journal of Surgery 2019; 25 (1):56-61.

15.Lin YB, Yu GS, Zhong ZH, et al. Biomechanical analysis of highly unstable femoral neck fractures fixed by four new configurations of cannulated nails. Chinese Journal of Traumatology and Orthopedics 2019; 
16.Chen DW, Xia H. Research progress of three hollow nails in treating femoral neck fractures. Chinese Journal of Physicians 2019; 21 (1):158-61.

17.Cheng PY. Finite element analysis of different numbers and arrangements of hollow lag screws for treatment of femoral neck fractures. Hebei Medical University, 2017 (Dissertation).

18.Wang YH, Guo XY. Analysis of clinical effect of three or four hollow nails in the treatment of patients with femoral neck fractures. The World 's Latest Medical Information Digest 2016; 16(87): 80.

19.Mo G. Explore the application of minimally invasive techniques in the treatment of femoral neck fractures with hollow nails. Zunyi Medical College, 2013 (Dissertation).

20.Hu WS, Li SZ, Yuan F. A comparative study of three and four hollow nails for the treatment of femoral neck fractures. Chinese Journal of Bone and Joint Injury 2013; 28 (4): 307-9.

21.Moher $D$, Shamseer $L$, Clarke $M$, et al. Preferred reporting items for systematic review and metaanalysis protocols (PRISMA-P) 2015 statement. Syst Rev 2015; 4:1.

22.Shamseer $L$, Moher $D$, Clarke $M$, et al. Preferred reporting items for systematic review and metaanalysis protocols (PRISMA-P) 2015: elaboration and explanation. BMJ 2015; 349:g7647.

23.Guyatt $\mathrm{GH}$, Oxman $\mathrm{AD}$, Vist $\mathrm{GE}$, et al. Grade: an emerging consensus on rating quality of evidence and strength of recommendations. BMJ 2008; 336:924-6.

24.Guyatt $\mathrm{GH}$, Oxman AD, Schünemann HJ, et al. Grade guidelines: a new series of articles in the Journal of clinical epidemiology. J Clin Epidemiol 2011; 64:380-2.

\section{Table}

Table 1 Search strategy utilized for MEDLINE database 


\begin{tabular}{ll}
\hline Number & Search terms \\
\hline 1 & femoral neck fractures \\
2 & hip fractures \\
3 & femoral head \\
4 & femoral neck \\
5 & femoral neck junction \\
6 & Or 1-5 \\
7 & four hollow nail \\
8 & rhombic fixation \\
9 & nail fixation \\
10 & fracture fixation \\
11 & Or 7-10 \\
12 & random \\
13 & randomly \\
14 & blind \\
15 & control \\
16 & comparator \\
17 & controlled trial \\
18 & clinical trial \\
19 & study \\
20 & Or 12-19 \\
21 & 6 and 11 and 20 \\
\hline
\end{tabular}

\section{Supplementary Files}

This is a list of supplementary files associated with this preprint. Click to download.

- PRISMAPchecklist.doc 